\title{
Radiative Decay Width of Neutral non-Strange Baryons from PWA
}

\author{
Igor I. Strakovsky, a William J. Briscoe ${ }^{1}$, Alexander E. Kudryavtsev ${ }^{1,2}$, Viacheslav V. Kulikov ${ }^{2}$, Maxim A. \\ Martemyanov $^{2}$ and Vladimir E. Tarasov ${ }^{2}$ \\ ${ }^{1}$ The George Washington University, Washington, DC 20052, USA \\ ${ }^{2}$ Institute for Theoretical and Experimental Physics, Moscow, 117218, Russia
}

\begin{abstract}
An overview of the GW SAID and ITEP groups effort to analyze pion photoproduction on the neutron-target will be given. The disentanglement the isoscalar and isovector EM couplings of $\mathrm{N}^{*}$ and $\Delta^{*}$ resonances does require compatible data on both proton and neutron targets. The final-state interaction plays a critical role in the state-of-the-art analysis in extraction of the $\gamma \mathrm{n} \rightarrow \pi \mathrm{N}$ data from the deuteron target experiments. It is important component of the current JLab, MAMI-C, SPring-8, ELSA, and ELPH programs.
\end{abstract}

\section{Introduction}

The $\mathrm{N}^{*}$ family of nucleon resonances has many well established members [1], several of which exhibit overlapping resonances with very similar masses and widths but with different $\mathrm{J}^{\mathrm{P}}$ spin- parity values. Apart from the $\mathrm{N}(1535) 1 / 2^{-}$state, the known proton and neutron photodecay amplitudes have been determined from analyses of single-pion photoproduction. The present work reviews the region from the threshold to the upper limit of the SAID analyses, which is CM energy $\mathrm{W}=2.5 \mathrm{GeV}$. There are two closely spaced states above $\Delta(1232) 3 / 2^{+}$: $\mathrm{N}(1520) 3 / 2^{-}$and $\mathrm{N}(1535) 1 / 2^{-}$. Up to $\mathrm{W} \sim 1800 \mathrm{MeV}$, this region also encompasses a sequence of six overlapping states: $\mathrm{N}(1650) 1 / 2^{-}, \mathrm{N}(1675) 5 / 2^{-}, \mathrm{N}(1680) 5 / 2^{+}$, $\mathrm{N}(1700) 3 / 2^{-}, \mathrm{N}(1710) 1 / 2^{+}$, and $\mathrm{N}(1720) 3 / 2^{+}$.

One critical issue in the study of meson photoproduction on the nucleon comes from isospin. While isospin can change at the photon vertex, it must be conserved at the final hadronic vertex. Only with good data on both proton and neutron targets can one hope to disentangle the isoscalar and isovector electromagnetic (EM) couplings of the various $\mathrm{N}^{*}$ and $\Delta^{*}$ resonances (see Refs. [2,3]), as well as the isospin properties of the non-resonant background amplitudes. The lack of $\gamma \mathrm{n} \rightarrow \pi^{-} \mathrm{p}$ and $\gamma \mathrm{n} \rightarrow \pi^{0} \mathrm{n}$ data does not allow us to be as confident about the determination of neutron EM couplings relative to those of the proton. For instance, the uncertainties of neutral EM couplings of $4^{*}$ low-lying $\mathrm{N}^{*}$ resonances, $\Delta\left(\mathrm{nA}_{1 / 2}\right)$ vary between 25 and $140 \%$ while charged EM couplings, $\Delta\left(\mathrm{nA}_{1 / 2}\right)$, vary between 7 and $42 \%$. Some of the $\mathrm{N}^{*}$ baryons $\left[\mathrm{N}(1675) 5 / 2^{-}\right.$, for instance] have stronger EM couplings to the neutron relative to the proton, but the parameters are very uncertain [1]. One more unresolved issue relates to the second $\mathrm{P}_{11}, \mathrm{~N}(1710) 1 / 2^{+}$. That is not seen in the recent $\pi \mathrm{N}$ PWA [4], contrary to other PWAs

\footnotetext{
${ }^{\mathrm{a} C}$ Corresponding author: igor@gwu.edu
}

used by the PDG14 [1]. A recent brief review of its status is given in Ref. [5]. Obviously, data on the $\gamma \mathrm{n} \rightarrow \pi \mathrm{N}$ reactions are needed to improve the amplitudes and EM couplings.

Additionally, incoherent pion photoproduction on the deuteron is interesting in various aspects of nuclear physics, and particularly, provides information on the elementary reaction on the neutron, i.e., $\gamma \mathrm{n} \rightarrow \pi \mathrm{N}$. Finalstate interaction (FSI) plays a critical role in the state-ofthe-art analysis of the $\gamma \mathrm{n} \rightarrow \pi \mathrm{N}$ interaction as extracted from $\gamma \mathrm{d} \rightarrow \pi \mathrm{NN}$ measurements. The FSI was first considered in Refs. [6,7] as responsible for the near-threshold enhancement (Migdal-Watson effect) in the NN mass spectrum of the meson production reaction $\mathrm{NN} \rightarrow \mathrm{NNx}$. In Ref. [8], the FSI amplitude was studied in detail.

\section{Complete Experiment in Pion Photoproduction}

Originally, PWA arose as the technology to determine amplitude of the reaction via fitting scattering data. That is a non-trivial mathematical problem - looking for a solution of ill-posed problem following to Hadamard, Tikhonov et al. Resonances appeared as a by-product (bound states objects with definite quantum numbers, mass, lifetime and so on).

There are 4 independent invariant amplitudes for a single pion photoproduction. In order to determine the pion photoproduction amplitude, one has to carry out 8 independent measurements at fixed $(\mathrm{W}, \mathrm{t})$ or $(\mathrm{E}, \theta)$ (the extra observable is necessary to eliminate a sign ambiguity [9]).

There are 16 non-redundant observables and they are not completely independent from each other, namely 1 unpolarized, $d \sigma / d \Omega ; 3$ single polarized, $\Sigma, \mathrm{T}$, and $\mathrm{P} ; 12$ double polarized, E, F, G, H, C ${ }_{x}, C_{z}, O_{x}, O_{z}, L_{x}, L_{z}, T_{x}$, and $\mathrm{T}_{\mathrm{z}}$ measurements. Additionally, there are 18 triple- 
polarization asymmetries [9 (9) for linear (circular) polarized beam and 13 of them are non-vanishing] [10,11]. Obviously, the triple-polarization experiments are not really necessary from the theoretical point of view while such measurements will play a critical role to keep systematics under control.

\section{Neutron Database}

Experimental data for neutron-target photoreactions are much less abundant than those utilizing a proton target, constituting only about $15 \%$ of the present worldwide known GW SAID database [12]. The existing $\gamma \mathrm{n} \rightarrow \pi^{-} \mathrm{p}$ database contains mainly differential cross sections and $15 \%$ of which are from polarized measurements. At low to intermediate energies, this lack of neutron-target data is partially compensated by experiments using pion beams, e.g., $\pi^{-} \mathrm{p} \rightarrow \gamma \mathrm{n}$, as has been measured, for example, by the Crystal Ball Collaboration at BNL [13] (the GW nuclear physics group was heavily involved in this experiment) for the inverse photon energy $\mathrm{E}=285-689 \mathrm{MeV}$ and $\theta=41^{\circ}-148^{\circ}$, where $\theta$ is the inverse production angle of $\pi^{-}$in the CM frame. This process is free from complications associated with the deuteron target. However, the disadvantage of using the reaction $\pi^{-} \mathrm{p} \rightarrow \gamma \mathrm{n}$ is the 5 to 500 times larger cross sections for $\pi^{-} \mathrm{p} \rightarrow \gamma \mathrm{n} \rightarrow \gamma \mathrm{nn}$, depending on $\mathrm{E}$ and $\theta$, which causes a large background, and there were no tagging high flux pion beams.

Figure 1 summarize the available data for single pion photoproduction on the neutron below $\mathrm{W}=2.5 \mathrm{GeV}$. Many high-precision data for the $\gamma \mathrm{n} \rightarrow \pi^{-} \mathrm{p}$ and $\gamma \mathrm{n} \rightarrow \pi^{0} \mathrm{n}$ reactions have been measured recently. We applied our GWITEP FSI corrections, covering a broad energy range up to $\mathrm{E}=2.7 \mathrm{GeV}$ [8], to the CLAS and A2 Collaboration $\gamma \mathrm{d} \rightarrow \pi^{-} \mathrm{pp}$ measurements to get elementary cross sections for $\gamma \mathrm{n} \rightarrow \pi^{-} \mathrm{p}[14,15]$. In particular, the new CLAS cross sections have quadrupled the world database for $\gamma \mathrm{n} \rightarrow \pi^{-} \mathrm{p}$ above $\mathrm{E}=1 \mathrm{GeV}$. The FSI correction factor for the CLAS $\left(\mathrm{E}=1050-2700 \mathrm{MeV}\right.$ and $\left.\theta=32^{\circ}-157^{\circ}\right)$ and MAMI $\left(\mathrm{E}=410-460 \mathrm{MeV}\right.$ and $\left.\theta=45^{\circ}-125^{\circ}\right)$ kinematics was found to be small, $\Delta \sigma / \sigma<10 \%$.

Obviously, that is not enough to have compatible proton and neutron databases, specifically the energy binning of the CLAS measurements is $50 \mathrm{MeV}$ or, in the worst case, $100 \mathrm{MeV}$ while A2 Collaboration measurements are able to have 2 to $4 \mathrm{MeV}$ binning. The forward direction, which is doable for A2 vs. CLAS, is critical for evaluation of our FSI treatment.

\section{Neutron Data from Deuteron Measurements}

The determination of the $\gamma \mathrm{d} \rightarrow \pi^{-}$pp differential cross sections with the FSI, taken into account (including all key diagrams in Fig. 2), were done, as we did recently $[8,14,15]$, for the CLAS [14] and MAMI data [15]. The SAID of GW Data Analysis Center (DAC) phenomenological amplitudes for $\gamma \mathrm{N} \rightarrow \pi \mathrm{N}$ [16], NN $\rightarrow \mathrm{NN}$ [17],
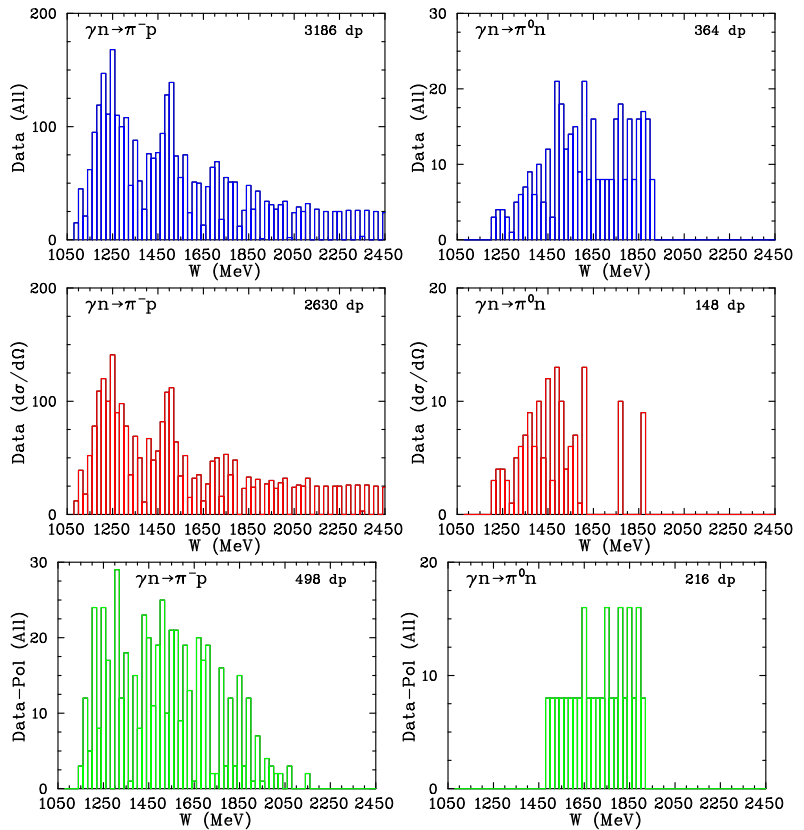

Figure 1. Data available for single pion photoproduction off the neutron as a function of CM energy W [12]. The number of data points, $\mathrm{dp}$, is given in the upper right hand side of each subplot. Left panels: The first subplot (blue) shows the total amount of $\gamma \mathrm{n} \rightarrow \pi^{-} \mathrm{p}$ data available for all observables, the second subplot (red) shows the amount of differential cross-section, $d \sigma / d \Omega$, data available, the third subplot (green) shows the amount of $\mathrm{P}$ observables data available. Right panels: The first subplot (blue) shows the total amount of $\gamma \mathrm{n} \rightarrow \pi^{0} \mathrm{n}$ data available for all observables, the second subplot (red) shows the amount of $d \sigma / d \Omega$ data available, the third subplot (green) shows the amount of $\mathrm{P}$ observables data available

and $\pi \mathrm{N} \rightarrow \pi \mathrm{N}$ [4] were used as inputs to calculate the diagrams in Fig. 2. The Bonn potential (full model) [18] was used for the deuteron description. In Ref. [14,15], we calculated the FSI correction factor R(E, $\theta)$ [Eq. (1)] dependent on photon energy, $\mathrm{E}$, and pion production angle in CM frame $\theta$ (see details in Refs. $[8,14,15]$ ) and fitted recent CLAS and MAMI $d \sigma / d \Omega$ versus the world $\gamma \mathrm{N} \rightarrow \pi \mathrm{N}$ database [12] to get new neutron multipoles and determine neutron resonance EM couplings [14].

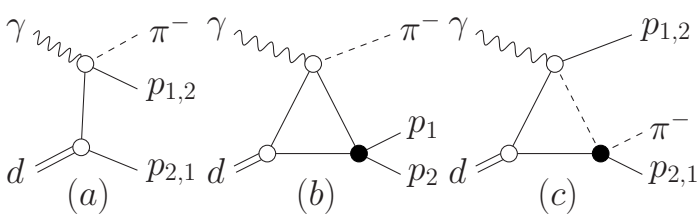

Figure 2. Feynman diagrams for the leading components of the $\gamma \mathrm{d} \rightarrow \pi^{-}$pp amplitude. (a) Impulse approximation (IA), (b) pp-FSI, and (c) $\pi$ N-FSI. Filled black circles show FSI vertices. Wavy, dashed, solid, and double lines correspond to the photons, pions, nucleons, and deuterons, respectively.

Results of calculations and comparison with the experimental data on the differential cross sections, $d \sigma_{\gamma \mathrm{d}} / d \Omega$, where $\Omega$ and $\theta$ are solid and polar angles of outgoing $\pi^{-}$ 
in the laboratory frame, respectively, with z-axis along the photon beam for the reaction $\gamma \mathrm{d} \rightarrow \pi^{-}$pp are given in Fig. 3 (upper panels) for a number of the photon energies, $\mathrm{E}$.
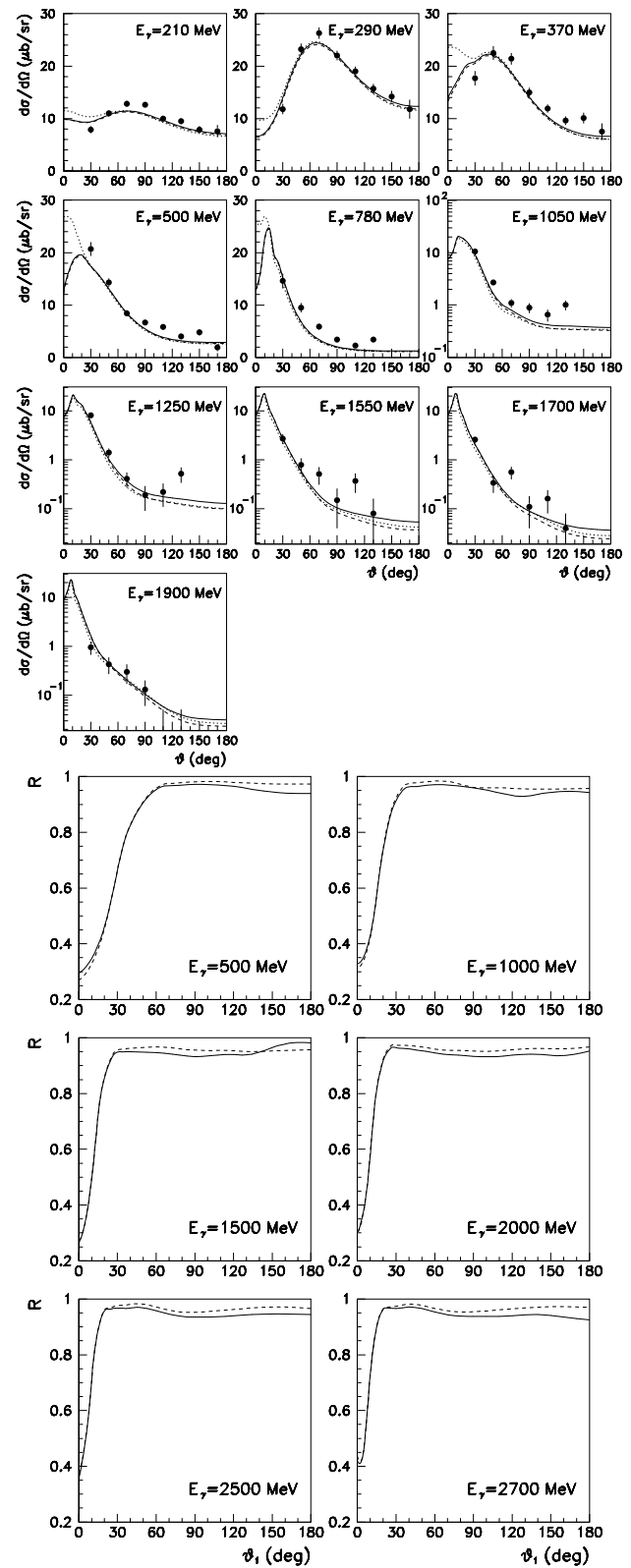

Figure 3. Upper panels: The differential cross section, $d \sigma_{\gamma \mathrm{d}} / d \Omega$, of the reaction $\gamma \mathrm{d} \rightarrow \pi^{-} \mathrm{pp}$ in the laboratory frame at different values of the photon laboratory energy $\mathrm{E}<1900 \mathrm{MeV}$; $\theta$ is the polar angle of the outgoing $\pi^{-}$. Dotted curves show the contributions from the IA amplitude [Fig. 2(a)]. Successive addition of the NN-FSI [Fig. 2(b)] and $\pi$ N-FSI [Fig. 2(c)] amplitudes leads to dashed and solid curves, respectively. The filled circles are the data from DESY bubble chamber [19]. Lower panels: The correction factor R, defined by Eq. (1), where $\theta$ is the polar angle of the outgoing $\pi^{-}$in the rest frame of the pair $\pi^{-}+$fast proton. The kinematic cut, $\mathrm{P}_{\mathrm{p}}>200 \mathrm{MeV} / \mathrm{c}$, is applied. The solid (dashed) curves are obtained with both $\pi \mathrm{N}$ - and NN-FSI (only NN-FSI) taken into account.

The FSI corrections for the CLAS and MAMI quasifree kinematics were found to be small, as mentioned above. Our FSI calculations were done $[8,14,15]$ over a broad energy range (threshold to $\mathrm{E}=2700 \mathrm{MeV}$ ) and for the full angular coverage $\left(\theta=0^{\circ}-180^{\circ}\right)$. As an illustration, Fig. 3 (lower panels) shows the FSI correction factor

$$
\mathrm{R}(\mathrm{E}, \theta)=\left(\mathrm{d} \sigma / \mathrm{d} \Omega_{\pi \mathrm{p}}\right) /\left(\mathrm{d} \sigma^{\mathrm{IA}} / \mathrm{d} \Omega_{\pi \mathrm{p}}\right)
$$

for the $\gamma \mathrm{n} \rightarrow \pi^{-} \mathrm{p}$ differential cross sections as a function of the pion production angle in the $\mathrm{CM}(\pi-p)$ frame, $\theta$, for different energies over the range of the CLAS and MAMI experiments. Overall, the FSI correction factor $\mathrm{R}(\mathrm{E}, \theta)<1$, while the effect, i.e., the $(1-\mathrm{R})$ value, vary from $10 \%$ to $30 \%$, depending on the kinematics, and the behavior is very smooth versus pion production angle. We found a sizeable FSI-effect from S-wave part of pp-FSI at small angles. A small but systematic effect $|R-1|<<1$ is found in the large angular region, where it can be estimated in the Glauber approach, except for narrow regions close to $\theta \sim 0^{\circ}$ or $\theta \sim 180^{\circ}$. The $\gamma \mathrm{n} \rightarrow \pi^{0} \mathrm{n}$ case is much more complicate vs. $\gamma \mathrm{n} \rightarrow \pi^{-}$p because $\pi^{0} n$ final state can come from both $\gamma \mathrm{n}$ and $\gamma \mathrm{p}$ initial interactions [20]. The leading diagrams for $\gamma \mathrm{d} \rightarrow \pi^{0} \mathrm{pn}$ are similar as given on Fig, 2.

\section{New Neutron Amplitudes and neutron EM Couplings}

The solution, SAID GB12 [14], uses the same fitting form as SAID recent SN11 solution [21], which incorporated the neutron-target CLAS $d \sigma / d \Omega$ for $\gamma \mathrm{n} \rightarrow \pi^{-} \mathrm{p}$ [14] and GRAAL $\Sigma$ s for both $\gamma \mathrm{n} \rightarrow \pi^{-} \mathrm{p}$ and $\gamma \mathrm{n} \rightarrow \pi^{0} \mathrm{n}[22,23]$ (Fig. 4). This fit form was motivated by a multichannel K-matrix approach, with an added phenomenological term proportional to the $\pi \mathrm{N}$ reaction cross section.

\begin{tabular}{|cc|ccc|c|}
\hline Resonance & $n A_{1 / 2}$ & Resonance & $n A_{1 / 2}$ & $n A_{3 / 2}$ & Ref. \\
\hline$N(1535) 1 / 2^{-}$ & $-58 \pm 6$ & $N(1520) 3 / 2^{-}$ & $-46 \pm 6$ & $-115 \pm 5$ & SAID GB12 \\
& $-60 \pm 3$ & & $-47 \pm 2$ & $-125 \pm 2$ & SAID SN11 \\
& $-93 \pm 11$ & & $-49 \pm 8$ & $-113 \pm 12$ & BnGa13 \\
& $-49 \pm 3$ & & $-38 \pm 3$ & $-101 \pm 4$ & Kent12 \\
$N(1650) 1 / 2^{-}$ & $-46 \pm 27$ & & $-59 \pm 9$ & $-139 \pm 11$ & PDG14 \\
& $-40 \pm 10$ & $N(1675) 5 / 2^{-}$ & $-58 \pm 2$ & $-80 \pm 5$ & SAID GB12 \\
& $-26 \pm 8$ & & $-42 \pm 2$ & $-60 \pm 2$ & SAID SN11 \\
& $25 \pm 20$ & & $-60 \pm 7$ & $-88 \pm 10$ & BnGa13 \\
$N(1440) 1 / 2^{+}$ & $-15 \pm 2$ & & $-40 \pm 4$ & $-68 \pm 4$ & Kent12 \\
& $48 \pm 4$ & $N(1680) 5 / 2^{+}$ & $-43 \pm 12$ & $-58 \pm 13$ & PDG14 \\
& $45 \pm 15$ & & $50 \pm 4$ & $-29 \pm 2$ & SAID GB12 \\
& $43 \pm 12$ & & $34 \pm 6$ & $-44 \pm 9$ & SAID SN11 \\
& $40 \pm 5$ & & $29 \pm 2$ & $-59 \pm 2$ & BnGa13 \\
& $40 \pm 10$ & & $29 \pm 10$ & $-33 \pm 9$ & PDG12 \\
\hline
\end{tabular}

Table 1. Neutron helicity amplitudes $A 1 / 2$ and A3/2 (in $[(\mathrm{GeV})-1 / 2 \times 10-3]$ units) from the SAID GB12 [14] (first row), previous SAID SN11 [21] (second row), recent BnGa13 by the Bonn-Gatchina group [25] (third row), recent Kent12 by the Kent State Univ. group [26] (forth row), and average values from the PDG14 [1] (fifth row).

However, these new CLAS cross sections departed significantly from our predictions at the higher energies, and greatly modified PWA result [14] (Fig. 4). Recently, the BnGa group reported a neutron EM coupling determination [25] using the CLAS Collaboration $\gamma \mathrm{n} \rightarrow \pi^{-} \mathrm{p}$ because $\pi^{0} n$ final state can come from both $\gamma \mathrm{n}$ and $\gamma \mathrm{p}$ initial interact $d \sigma / d \Omega$ with our FSI [14] (Table 1). BnGa13 and 

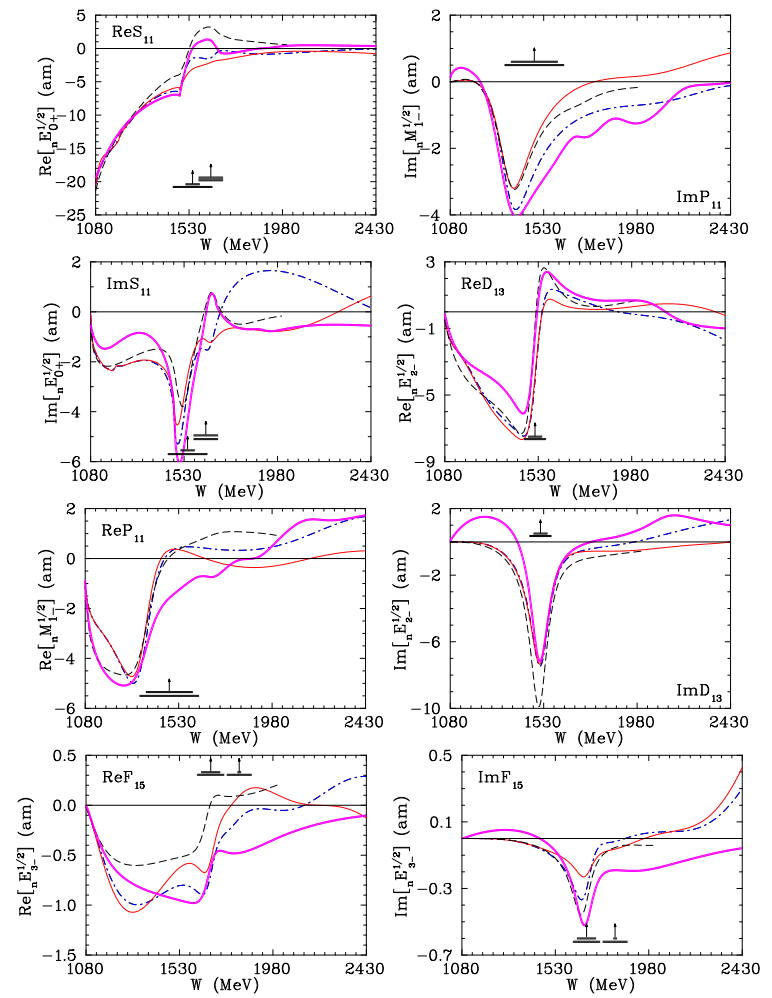

Figure 4. Samples of neutron multipoles $I=1 / 2$ and $3 / 2$. Solid (dash-dotted) lines correspond to the SAID GB12 [14] (SN11 [21]) solution. Thick solid (dashed) lines give SAID GZ12 [14] solution (MAID07 [24], which terminates at $\mathrm{W}=2 \mathrm{GeV}$ ). Vertical arrows indicate mass (WR), and horizontal bars show full, $\Gamma$, and partial, $\Gamma_{\pi \mathrm{N}}$, widths of resonances extracted by the BreitWigner fit of the $\pi \mathrm{N}$ data associated with the SAID solution WI08 [4].

SAID GB12 used the same (almost) data [14] to fit them while BnGa13 has several new Ad hoc resonances.

Overall: the difference between MAID07 with BnGa13 and SAID GB12 is rather small but resonances may be essentially different (Table 1). The new BnGa13 [25] has some difference vs. GB12 [14], PDG14 [1], for instance, for $\mathrm{N}(1535) 1 / 2^{-}, \mathrm{N}(1650) 1 / 2^{-}$, and $\mathrm{N}(1680) 5 / 2^{+}$.

\section{Work in Progress}

At MAMI in March of 2013, we collected deuteron data below $\mathrm{E}=800 \mathrm{MeV}$ with $4 \mathrm{MeV}$ energy binning [27] and will have a new experiment below $\mathrm{E}=1600 \mathrm{MeV}$ [28] in spring of 2015.

The experimental setup provides close to $4 \pi$ sr coverage for outgoing particles [Fig. 5 (top)]. The photons from $\pi^{0}$ decays and charged particles are detected by the CB and TAPS detection system. The energy deposited by charged particles in CB and TAPS is, for the most part, proportional to their kinetic energy, unless they punch through crystals of the spectrometers. Clusters from the final-state neutrons provide information only on their angles. Separation of clusters from neutral particles and charged ones is based on the information from MWPC, PID, and TAPS veto. Separation of positive and negative pions can be based on the identification of the final-state nucleon as either a neutron or a proton. Since cluster energies from charged pions are proportional to their kinetic energy (unless their punch through the crystals), the energy of those clusters can be very low close to reaction threshold.
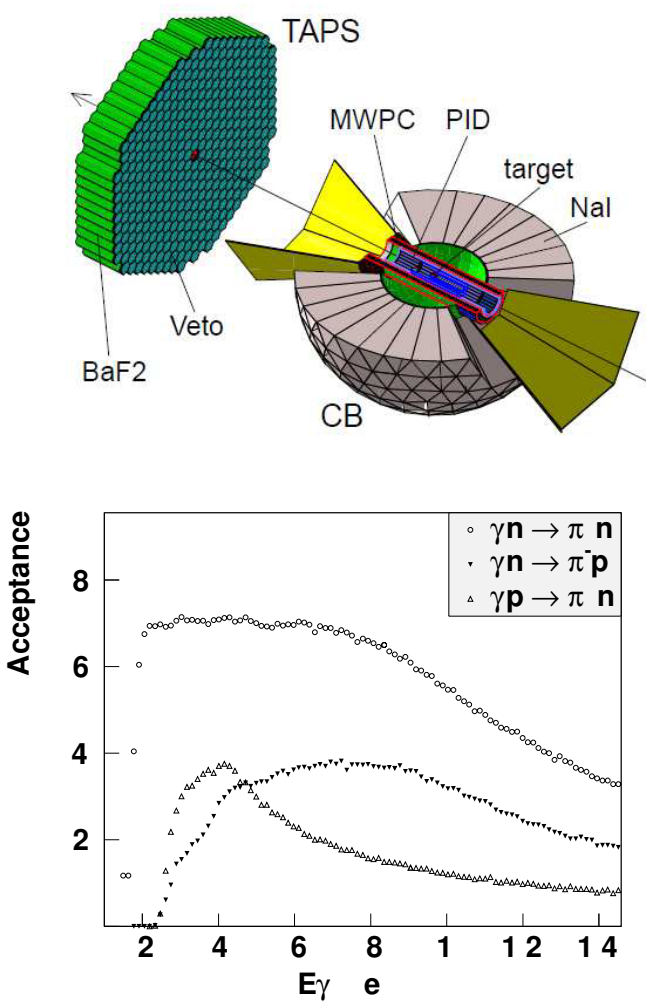

Figure 5. Top: The A2 detector setup: the Crystal Ball multiphoton spectrometer, with cut-away section showing the inner detectors, and the TAPS forward wall. Bottom: Average acceptance for three reactions $\gamma \mathrm{n} \rightarrow \pi^{0} \mathrm{n}, \gamma \mathrm{n} \rightarrow \pi^{-} \mathrm{p}$, and $\gamma \mathrm{p} \rightarrow \pi^{+} \mathrm{n}$ with the proposed trigger [28].

Monte Carlo simulations [29], which tracks reaction products through a realistic model of the detector system together with the reconstruction program, is used to calculate acceptance to various channels [Fig. 5 (top)]. So to detect the reactions under study with our setup, we have to take data with almost open trigger. In Fig. 5 (bottom), we illustrate the average acceptance obtained for three reactions by requiring the total energy deposited in the $\mathrm{CB}$ to be larger than $100 \mathrm{MeV}$. As seen, acceptance for reaction $\gamma \mathrm{n} \rightarrow \pi^{0} \mathrm{n}$ varies from $70 \%$ at $0.8 \mathrm{GeV}$ to $30 \%$ at 1.5 $\mathrm{GeV}$ of the incident-photon energy. Acceptance of reaction $\gamma \mathrm{p} \rightarrow \pi^{+} \mathrm{n}$ drops at higher beam energies as charged pions punch through the crystals, and the energy of the neutron cluster does not reflect its kinetic energy. Reaction $\gamma \mathrm{n} \rightarrow \pi^{-} \mathrm{p}$ above $0.8 \mathrm{GeV}$ has an acceptance that is better than that for $\gamma \mathrm{p} \rightarrow \pi^{+} \mathrm{n}$ as the energy and angles of the cluster from the outgoing proton can be used to reconstruct the reaction kinematics.

We are going to use our FSI technology to apply for the upcoming JLab CLAS (g13 run period) $d \sigma / d \Omega$ for $\gamma \mathrm{n} \rightarrow$ 
$\pi^{-} \mathrm{p}$ covering $\mathrm{E}=400-2500 \mathrm{MeV}$ and $\theta=18^{\circ}-152^{\circ}$ [30]. This data set will bring about $12 \mathrm{k}$ new measurements which quadruple the world $\gamma \mathrm{n} \rightarrow \pi^{-} \mathrm{p}$ database. The ELPH facility at Tohoku Univ. will bring new $d \sigma / d \Omega$ for $\gamma \mathrm{n} \rightarrow \pi^{0} \mathrm{n}$ below $\mathrm{E}=1200 \mathrm{MeV}$ [31].

\section{Polarized Measurements}

The difference between previous pion photoproduction and new polarized measurements may result in significant changes in the neutron EM couplings. The strategy for the FSI calculations addressed to $\Sigma\left(\gamma \mathrm{d} \rightarrow \pi^{-} \mathrm{pp}\right)$ to get $\Sigma\left(\gamma \mathrm{n} \rightarrow \pi^{-} \mathrm{p}\right)$ is under consideration now. Our preliminary conclusion is that the FSI correction for the $\Sigma$-beam asymmetry is small far away from the pion threshold.

The SAID SP09 solution [32] is consistent with the GRAAL $\Sigma$-data for $\gamma \mathrm{n} \rightarrow \pi^{-} \mathrm{p}$ in the forward angular region where previous results constrained the fit (Fig. 6). In the backward region and at energies above $1100 \mathrm{MeV}$, the agreement becomes satisfactory only after inclusion of GRAAL data. The MAID2007 [24] solution agrees with GRAAL data in the forward region. Both SAID-SP09 and MAID2007 results exhibit structures not seen in the data and which explain the poor $\chi^{2}$ for both cases.

The upcoming JLab CLAS (g13 run period) $\Sigma$-data for $\gamma \mathrm{n} \rightarrow \pi^{-} \mathrm{p}$, covering $\mathrm{E}=910-2394 \mathrm{MeV}$ and $\theta=18^{\circ}-$ $131^{\circ}$, [33] have small disagreement with recent GRAAL measurements [22].

Previous $\gamma \mathrm{n} \rightarrow \pi^{-} \mathrm{p}$ measurements provided a better constraint vs. $\gamma \mathrm{n} \rightarrow \pi^{0}$ ncase. 216 new GRAAL $\Sigma$ s for $\gamma \mathrm{n} \rightarrow \pi^{0} \mathrm{n}$ are $60 \%$ of the world $\pi^{0} \mathrm{n}$ data (Fig. 7). For the GRAAL $\gamma \mathrm{n} \rightarrow \pi^{0} \mathrm{n}\left(\gamma \mathrm{n} \rightarrow \pi^{-} \mathrm{p}\right)$, the SAID SP09 had $\chi^{2} /$ data $=223$ (89) while SAID MA09 got $\chi^{2} /$ data $=3.1$ (4.9). SAID MA09 included both GRAAL $\Sigma$ measurements [22,23].

\section{Summary for Neutron Study}

- The differential cross section for the processes $\gamma \mathrm{n} \rightarrow$ $\pi^{-} \mathrm{p}$ was extracted from new CLAS and MAMI-B measurements accounting for Fermi motion effects in the IA as well as $\mathrm{NN}$ - and $\pi \mathrm{N}$-FSI effects beyond the IA.

- Consequential calculations of the FSI corrections, as developed by the GW-ITEP Collaboration, was applied.

- New cross sections departed significantly from our predictions, at the higher energies, and greatly modified the fit result.

- New $\gamma \mathrm{n} \rightarrow \pi^{-} \mathrm{p}$ and $\gamma \mathrm{n} \rightarrow \pi^{0} \mathrm{n}$ data will provide a critical constraint on the determination of the multipoles and EM couplings of low-lying baryon resonances using the PWA and coupled channel techniques.

- Polarized measurements at JLab/JLab12, MAMI, SPring-8, ELSA, and ELPH will help to bring more physics in.

- FSI corrections need to apply.

\section{Acknowledgements}

This material is based upon work supported by the U.S. Department of Energy, Office of Science, Office of Nuclear Physics, under Award Number DE-FG02-99ER41110. AK, MM, VK, and VT thank the Institute for Kernphysik at Mainz where part of this work was performed for hospitality and support.

\section{References}

[1] K.A. Olive et al. (Particle Data Group), Chin. Phys. C 38, 090001 (2014); http://pdg.lbl.gov/.

[2] K.M. Watson, Phys. Rev. 95, 228 (1954).

[3] R.L. Walker, Phys. Rev. 182, 1729 (1969).

[4] R.A. Arndt, W.J. Briscoe, I.I. Strakovsky, and R.L. Workman, Phys. Rev. C 74, 045205 (2006).

[5] Ya I. Azimov and I.I. Strakovsky, Proceedings of the XVth International Conference on Hadron Spectroscopy (Hadron 2013), Nara, Japan, Nov. 2013, PoS (Hadron 2014) 034.

[6] A.B. Migdal, JETP 1, 2 (1955).

[7] K.M. Watson, Phys. Rev. 88, 1163 (1952).

[8] V.E. Tarasov, W.J. Briscoe, H. Gao, A.E. Kudryavtsev, and I.I. Strakovsky, Phys. Rev. C 84, 035203 (2011).

[9] G. Keaton and R.L. Workman, Phys. Rev. C 54, 1437 (1996).

[10] A.M. Sandorfi, B. Dey, A. Sarantsev, L. Tiator, and R. Workman, AIP Conf. Proc. 1432, 219 (2012).

[11] K. Nakayama, private communication, 2014.

[12] W.J. Briscoe, D. Schott, I.I. Strakovsky, and R.L. Workman, Institute of Nuclear Studies of The George Washington University Database; http://gwdac.phys.gwu.edu/analysis/pr_analysis.html .

[13] A. Shafi et al, Phys. Rev. C 70, 035204 (2004).

[14] W. Chen, H. Gao,W.J. Briscoe, D. Dutta, A.E. Kudryavtsev, M. Mirazita, M.W. Paris, P. Rossi, S. Stepanyan, I.I. Strakovsky, V.E. Tarasov, and R.L. Workman, Phys. Rev. C 86, 015206 (2012).

[15] W.J. Briscoe, A.E. Kudryavtsev, P. Pedroni, I.I. Strakovsky, V.E. Tarasov, and R.L. Workman, Phys. Rev. C 86, 065207 (2012).

[16] M. Dugger, J.P. Ball, P. Collins, E. Pasyuk, B.G. Ritchie, R.A. Arndt, W.J. Briscoe, I.I. Strakovsky, R.L. Workman et al. (CLAS Collaboration), Phys. Rev. C 76, 025211 (2007).

[17] R.A. Arndt, W.J. Briscoe, I.I. Strakovsky, and R.L.Workman, Phys. Rev. C 76, 025209 (2007).

[18] R. Machleidt, K. Holinde, and C. Elster, Phys. Rep. 149, 1 (1987).

[19] P. Benz et al. (Aachen-Bonn-Hamburg-HeidelbergMuenchen Collaboration), Nucl. Phys. B 65, 158 (1973).

[20] V. Tarasov, A. Kudryavtsev, W. Briscoe, M. Dieterle, B. Krusche, I. Strakovsky, and M. Ostrick, in progress.

[21] R.L. Workman, W.J. Briscoe, M.W. Paris, and I.I. Strakovsky, Phys. Rev. C 85, 025201 (2012). 
[22] G. Mandaglio et al. (GRAAL Collaboration), Phys. Rev. C 82, 045209 (2010).

[23] R. Di Salvo et al. (GRAAL Collaboration), Eur. Phys. J. A 42, 151 (2009).

[24] D. Drechsel, S.S. Kamalov, and L. Tiator, Eur. Phys. J. A 34, 69 (2007); http://www.kph.unimainz.de/MAID/ .

[25] A. Anisovich V. Burkert, E. Klempt, V.A. Nikonov, A.V. Sarantsev, and U. Thoma, Eur. Phys. J. A 49, 67 (2013).

[26] M. Shrestha and D.M. Manley, Phys. Rev. C 86, 055203 (2012).

[27] Meson production off the deuteron. II, Spokespersons: W.J. Briscoe, V.V. Kulikov, K. Livingston, and I.I. Strakovsky (A2 Collaborations), MAMI Proposal MAMI-A2-02/13, Mainz, Germany, 2013.

[28] Meson production off the deuteron. I, Spokespersons: W.J. Briscoe and I.I. Strakovsky (A2 Collaborations), MAMI Proposal MAMI-A2-02/12, Mainz, Ger- many, 2012.

[29] D.I. Glazier, A Geant4 Simulation of the Crystal Ball@MAMI Setup, 2012; http://www.ph.ed.ac.uk/nuclear/G4/ .

[30] P. Mattione, Proceedings of the XVth International Conference on Hadron Spectroscopy (Hadron 2013), Nara, Japan, Nov. 2013, PoS (Hadron 2014) 096.

[31] T. Ishikawa et al., Proceedings of the XVth International Conference on Hadron Spectroscopy (Hadron 2013), Nara, Japan, Nov. 2013, PoS (Hadron 2014) 095.

[32] M. Dugger, J.P. Ball, P. Collins, E. Pasyuk, B.G. Ritchie, R.A. Arndt, W.J. Briscoe, I.I. Strakovsky, R.L. Workman et al. (CLAS Collaboration), Phys. Rev. C 79, 065206 (2009).

[33] G.Y. Chen, S.S. Kamalov, S.N. Yang, D. Drechsel, and L. Tiator, Phys. Rev. C 76, 035206 (2007).

[34] D. Sokhan, PhD Thesis, University of Edinburgh, 2009. 


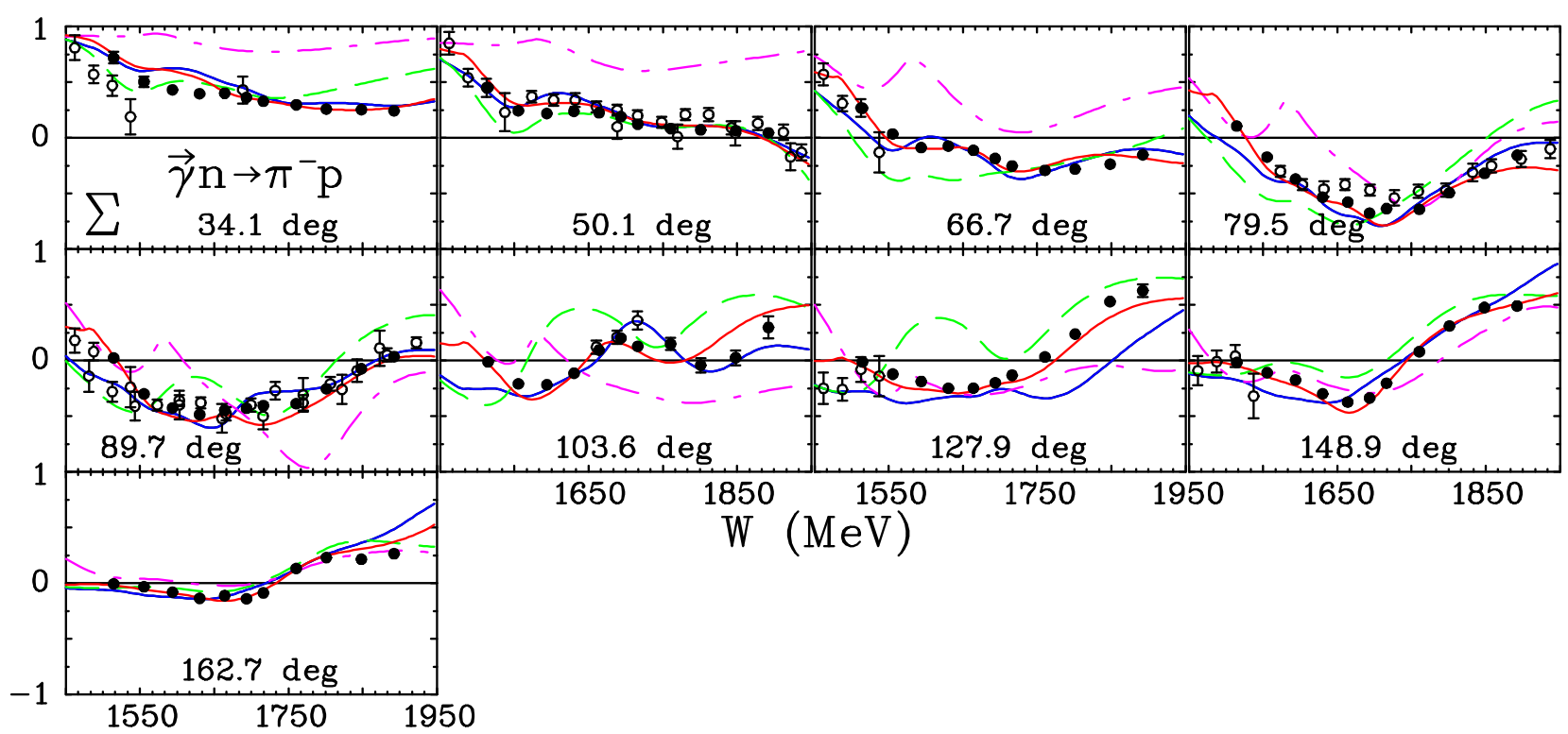

Figure 6. The $\Sigma$-beam polarization asymmetries for $\gamma \mathrm{n} \rightarrow \pi^{-} \mathrm{p}$ versus CM energy W. The pion CM production angle is shown. Solid red (solid blue) lines correspond to the SAID MA09 [22] (SP09 [32]) solution. Green dashed (magenta dot-dashed) lines give the MAID2007 [24] (DMT [33]) predictions. Experimental data are from the GRAAL [22] (filled circles) and previous measurements [12] (open circles). The plotted points from previously published experimental data are those data points within $4 \mathrm{MeV}$ of the photon energy indicated on each panel. Plotted uncertainties are statistical. The MA09 includes in its database the GRAAL asymmetries for $\gamma \mathrm{n} \rightarrow \pi^{-} \mathrm{p}[22]$ and $\gamma \mathrm{n} \rightarrow \pi^{0} \mathrm{n}[23]$ reactions. SP09 and MAID2007 do not include these $\Sigma$ data.

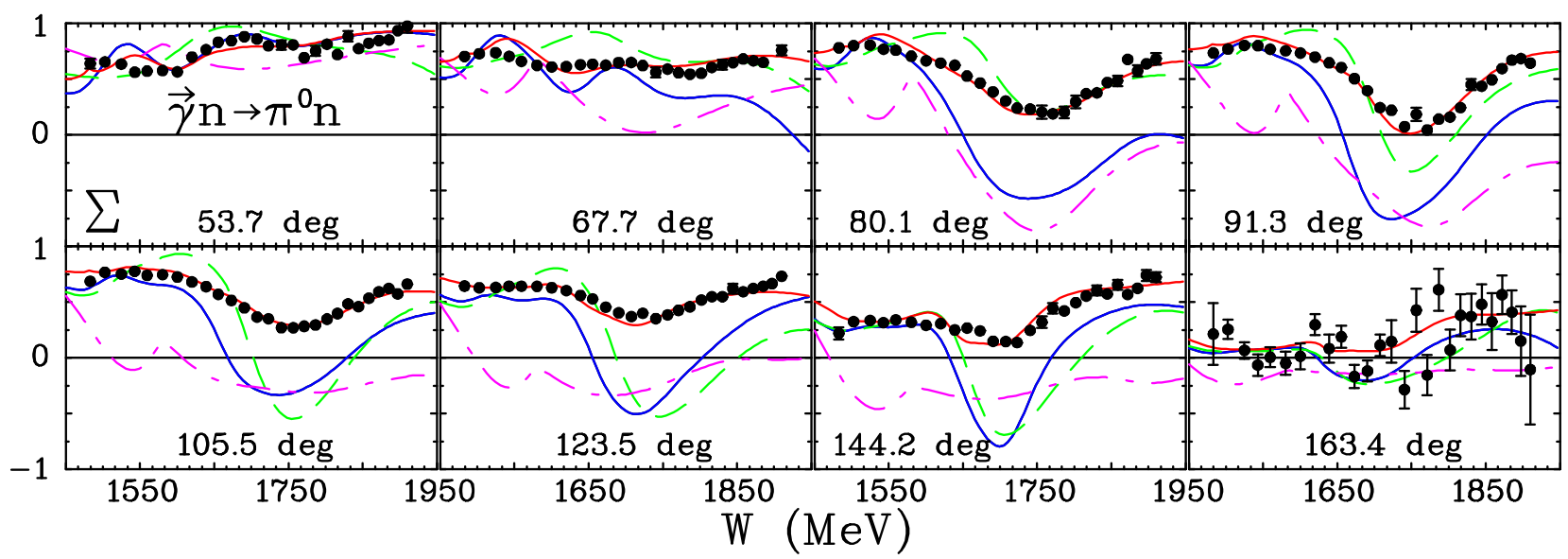

Figure 7. The $\Sigma$-beam polarization asymmetries for $\gamma \mathrm{n} \rightarrow \pi^{0} \mathrm{n}$ versus CM energy W. Notation as in Fig. 6 . 